\title{
Efficient Acoustic Communication Techniques for Nanobots
}

\author{
Valeria Loscrí \\ University of Calabria \\ Cosenza, Italy \\ vloscri@deis.unical.it
}

\author{
Valentina Mannara \\ University of Calabria \\ Cosenza, Italy \\ vmannara@deis.unical.it \\ Gianluca Aloi \\ University of Calabria \\ Cosenza, Italy \\ aloi@deis.unical.it
}

\author{
Enrico Natalizio \\ INRIA POP \\ Lille, France \\ enrico.natalizio@inria.fr
}

\begin{abstract}
In this work we present the simulation of a swarm of nanobots that behave in a distributed fashion and communicate through vibrations, allowing a decentralized control to treat endogenous diseases of the brain. Each nanobot is able to recognize a cancer cell, eliminate it and announce through a communication based on acoustic signals the presence of the cancer to the other nanobots. Acoustic communication technique is supported from the use of many diagnostic techniques for many years that did not have negative collateral side effects. We propose an approach based on the bees behavior in order to allow our devices to communicate, coordinate and reach the common objective to destroy the cancerous cells. In order to evaluate the effectiveness of our technique, we compared it with another technique known in literature and simulation results showed the effectiveness of our technique both in terms of achievement of the objective, that is the destruction of the cancerous cells, and the velocity of destruction.
\end{abstract}

\section{Categories and Subject Descriptors}

C.2.1 [Computer-Communication Networks]: Network Architecture and DesignDistributed Networks, Wireless Communication

\section{Keywords}

Nanomedicine, Brain tumor, swarm, acoustic communication

\section{INTRODUCTION}

Nanotechnologies are a new approach based on comprehension and deep knowledge of the properties of the matter at the nanoscale level: a nanometer corresponds to the length of a small molecule. Bio-medical field could be literally rev- olutionized from the potential applications of nano-devices, both in the diagnostic and pharmaceutic fields [5]. One of the most interesting applications would be to study the controlled release of drugs over time and exactly localized in cells or organs that need it, drastically reducing the side effects. Among the first applications to be postulated in the early ' 90 s and also among the most fascinating of nanomedicine is the idea of using nanorobots [1]. A device of a few nanometers (nanorobots will typically be 0.5 to 3 microns large with 1-100 nm parts), could be introduced into the body without causing injury and, if equipped with sensors that transmit precise images could facilitate the early diagnosis of cancer and carry drug to the target or to perform other tasks that would otherwise require invasive surgery [2]. A nanorobot in vivo, will prevent itself from being attacked by the immune system by diamond exterior will have to be smooth and flawless because this prevents Ieukocytes activities since the exterior is chemically inert and have low bioactivity [3]. The very limited size of the devices implies limited capabilities and reduced computation resources and for that it is necessary to make them collaborate by applying design techniques known as Swarm Intelligence in order to realize complex systems through the interaction and the cooperation of very simple agents [4].

This work is inspired by the paper [7]. The objective of authors was the evaluation of a swarm of nanobots, organized without any centralized unit, only trough simple local rules, to destroy cancer cells inside the brain.

The Lewis's work [7] has been an inspiration source for other works and represents one of the early works where it is assumed the use of robots for in vivo applications through a swarm technique. In [7] the type of communication proposed is a chemical communication, that is, the reporting of either some events or object is realized through the release of chemical substances that diffuse by attracting the other nanorobots through the gradient associated to the signal intensity. Lewis' strategy is based on three chemical substances that attract in a gradual fashion the swarm of nanobots to destroy diseased cells. An early part of our work was devoted to the tuning of the characteristic parameters of the Lewis' algorithm where objective difficulties emerged, related to the extremely reduced dimensions of the devices that do not allow to have computational capabilities 
and structural space needed to install the sensors and the interfaces to capt all the chemical substances involved. For these reasons we considered a different approach inspired from the bees behaviors that use vibration to communicate and self-organize. The rest of the paper is structured as follows. In Section II we present the problem. In Section III we give some details about the Lewis's approach. In Section IV we describe our bee's approach. In Section V we give the details about the simulation environment and results of Simulation. Finally we conclude the paper and we give some directions for future work in Section VI.

\section{PROBLEM DEFINITION}

The problem we consider in this paper is the presence of tumor cells in a human brain. The brain is represented as a field of cells. A single cell is the measure unit of the space and only one nanobot at a time can occupy a single cell. Inside the grid the cancer is positioned with the colony of nanobots, each of them is able to move step by step in one of the 8 adjacent cells. The goal is the elimination of all the cancer cells. Nanorobots move randomly in the field, even if we took into account the Brownian motion, since we assume our devices move into the blood. From a computational point of view, our devices are very simple since our algorithm only requires that each device has capabilities of:

- recognize a cancer cell;

- destroy a cancer cell;

- emit vibrations to signal the position of the cancer;

- detect acoustic waves.

\section{THE LEWIS ALGORITHM}

The Lewis' technique is based on chemical communication. Each $\mu$ - robot is able to mark its surrounding through chemical substances, recognizes the different chemical signals and follows the different gradients until it reaches the cancer cells. The colony is injected close to the cancer. Once injected into the body, the $\mu$-robots move randomly until they reach cancer cells. After the first contact the $\mu-$ robot emits in its surrounding a substance called CHEM-1, that is absorbed by the body after a certain time. A certain percentage of $\mu$-robots differentiate in guidepost, stop and start to secrete substances that allow the transmission of the signal over long distances. These substances are CHEM-2 and CHEM-3 and are used such as repeaters. The number of $\mu$-robots that differentiate in guideposts determine the efficiency, that is the convergence of $\mu$-robots to defeat the tumor. The number of guideposts is very important. In fact, if too many nanobots differentiate it is difficult to reach and destruct the tumor. On the other hand, if the number of guideposts is too low, the colony is not able to arrange. It was for that the authors considered a differentiation probability $p=0.01$ and related the total number of guideposts to the total number of $\mu$-robots $n$ and the current time $t$, pnt.

The algorithm's efficiency is strictly related to some additional parameters, i.e. $\Theta$, that represents a threshold value (the concentration of CHEM-1) that we analyzed. The environment considered is the cerebral cortex that is represented in the simulator NSl [8] as a cells array. The modeled chemical communication takes into account the generation, the diffusion of the substances immersed in a fluid and the absorption factor. Once a $\mu-$ robot reaches a cancer cell is able to perceive the tumor and to signal to other the presence of the cancer. The tumor is constituted by 42 cells. We adopted two different convergence criteria. The first one is represented by the total elimination of the cancer cells and the other is a number of 1000 iterations. We estimated that after 1000 iterations nodes come back to the same positions without be able to eliminate other cancer cells.

\section{THE BEES APPROACH}

Our nanobots borrow from the bees the capabilities to communicate through vibration (waggle dance for the bees). The auto-organization of the bees is based on very simple rules related to the behavior of each individual. We refer to this bee's inspired technique as NanoBee and is supported by the possibility of using acoustic waves as transmission means in communications in vivo without specific risks associated to. We exploited the analysis made in [5] to characterize our simulation model and the tuning of our parameters. The vibrations associated to the devices generate acoustic waves that propagate in an elastic medium and can be detected from a acoustic detector. Sound can be indifferently expressed as Sound Power, Sound Intensity or Sound Pressure. Sound Power is the total amount of acoustic energy emitted from a sound source and is measured in watt. Sound Intensity is the ratio of the power of a sound wave and the crossed superficial area, it is usually measured in watt $/$ meter $^{2}$. Sound Pressure is the value of the pressure variation of a corps in a generic point inside the sound field and is measured in newton $/$ meter $^{2}$. For sake of simplicity we assume sound that propagates freely without any obstacle. With this assumption and when the medium considered is non-dissipative, Intensity (I), power (W) and pressure (p) are correlated in this way:

$$
I=\frac{W}{2 \pi r^{2}}=\frac{p^{2}}{\rho c}
$$

where $\rho$ is the density of the fluid and $\mathrm{c}$ is the light speed. It is worth to note that both intensity and pressure decrease with the square of the distance (r). Based on these considerations we assumed the possibility of designing a technique based on acoustic communication by modeling the propagation of signals with Sound Intensity. NanoBee technique considers nanobots as point sources, since the dimensions are small compared to the distance from the receiver. Each nanobot is able to signal the presence of a cancer cell by stopping its movement and starting to vibrate, mimicking the bees behavior, in order to transmit a sound signal to alert the other agents (nanobots). Every nanobot receiving the signal moves towards the gradient of the intensity received. Vibrating time is limited since it depends on the force associated to each device to accomplish this vibration movement. In order to simulate the spatial variation of Sound Intensity, we assumed three different probability intervals, that simulate both the spatial propagation and the attenuation of the sound. Specifically, we choose higher probabilities values close to the nanobot that is vibrating and probabilities 
values smaller when distances are greater by simulating in this way the attenuation with the square of the distance. A more realistic version of the algorithm should take into account the temporal attenuation of a sound signal and in this case there is a decreasing of both the intensity and the distance reached from the signal. The first version, where we do not take into account the temporal attenuation of the signal is referred as NanoBee ON-OFF, where ON and OFF indicate that whether the device is "ON", it vibrates with the maximum power otherwise it is "OFF" and does not vibrate at all. The second version of our algorithm is referred as NanoBeeEvan and is related with a temporal attenuation of the sound signal. To the follow we give the pseudo-code of the NanoBee:

\section{Algorithm 2 NanoBee}

\section{Repeat}

FOR each "active" nanobot $i$ :

pick up any sound signal in its surrounding;

IF there are not any signal/cancer cells

search randomly;

IF discovered a cancer cell

THEN eliminate the cell and starts to dancing;

IF a signal has been received

THEN moves towards the higher intensity of the signal;

Until there is an "active" nanobot

A nanobot is active when it has enough energy, above a certain predetermined threshold and it did not discover yet any cancer cell.

\section{SIMULATION RESULTS}

We simulated the systems previously described with Neural Simulation Language (NSL) [8]. NSL possesses many features which facilitated simulation development, including graphics capability, and language constructs for handling layers of grid object and was also considered from [7].

The space where nanobots move and act is a cells grid modeling the vertebral cortex. We considered 42 cancer cells in every configuration and 289 nanobots in according to [7]. The effectiveness of all techniques considered in this work was evaluated by considering the time needed to defeat cancer cells and the capability to defeat all the tumor.

The parameters we considered in order to evaluate the performance of the various techniques are summarized in table 1. The algorithms considered in this work are based on the concept of nanobots swarm, that allows us to consider a reduced dimension of devices with available power smaller than that necessary if only a device is considered as observed in [6]. Frequencies ranging from 1 to $5 \mathrm{MHz}$ are typically used for head [6] and for this reason we choose the value of frequency equal to $1 \mathrm{MHz}$. Moreover, in our bee approach, communication activity is only related to the achievement phase of the target. In practice, nanobots start to vibrate when they discover a tumor and not when they move. We applied the method of Independent Replications with a confidence interval of $95 \%$.
Table 1: Simulation Parameters

\begin{tabular}{|c||c|}
\hline Searching Space & $50 \times 50$ cells \\
\hline \# Nanobots & 289 \\
\hline Cancer size & 42 cells \\
\hline Size of nanobots & $0.5 \mu \mathrm{m}$ \\
\hline Frequency & $10 \mathrm{MHz}$ \\
\hline Power & $0.5 \mathrm{pW}$ \\
\hline Simulation Time & $1000 \mathrm{runs}$ \\
\hline Prob Values & {$[1-0.7],[0.69-0.3],[0.29,0]$} \\
\hline Confidential Interval & $95 \%$ \\
\hline
\end{tabular}

In Fig. 1 we show the convergence time of each algorithm considered. We can notice that NanoLewis approach is not able to destroy all the cancer cells even if we consider the maximum number of nanobots (289). We conducted a sensitivity analysis to set parameters of Lewis' approach and we chose the set of parameters that guarantees the best performance in terms of convergence. Anyway, during the simulation we observed as the combination of the three chemical substances determines the formation of a kind of "barrier effect". In practice, after the first nanobots enter in contact with cancer cells and a part of the devices differentiate and start to work as relay by sending CHEM-1, for the other nanobots is very difficult to attack the most internal cancer cells and when the stations differentiate like "guidepost" and start to send CHEM-2 if they already entered in contact with CHEM-1 or CHEM-3 if they were touched from CHEM-2, the situation worsens. Bee's approaches behave similarly with the difference that NanoBee ON-OFF is able to eliminate all the cancer cells with less nanobots than NanoBeeEvan. NanoBee ON-OFF is more effective in this case thanks to the signal propagation that reaches farther during the time and is able to "attract" more nanobots. NanoBeeEvan is characterized with the attenuation of the signal power during the time and this means that after a certain period the signal only reaches a shorter distance.

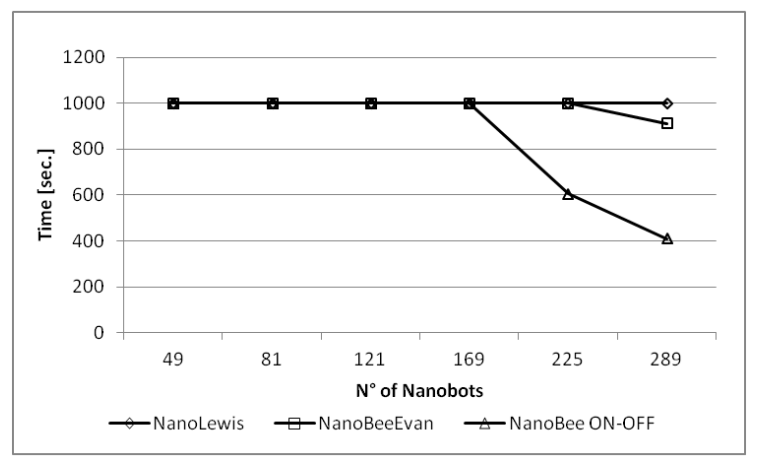

Figure 1: Convergence Time.

It is worth to note how, both the nano-bee approaches require less nanobots to reach the goal to eliminate all the cancer cells than those considered in Lewis paper. In our 
opinion the analysis of the performance in terms of both effectiveness, that is the capability to destroy all the cancer cells, and the rapidity to do it by varying the number of nanobots, is very important because very important is to use the minimum number of nanobots in a similar context. In fact, we have to recall that we are considering the human brain and the vessels that carry out both the data and the nanobots are very thin. Moreover, it is very important to try to reduce as much as possible both the chemical and the acoustic messages.

The other parameter we considered for our analysis is the amount of eliminated cells. In Fig. 2 we can observe how the number of cancer cells eliminated by the NanoLewis technique is always smaller than the other two techniques. As already outlined before, we observed that it is effective when the first nanobots attach the tumor, but the barrier created through the chemical substances does not allow to penetrate inside the "heart" of cancer. In fact, even if the number of nanobots increases, the effect does not change. This shows that the scarce efficiency is not related to the small number of nanobots. On the contrary, bees techniques are more able to defeat cancer cells when an higher number of nano-devices is considered.

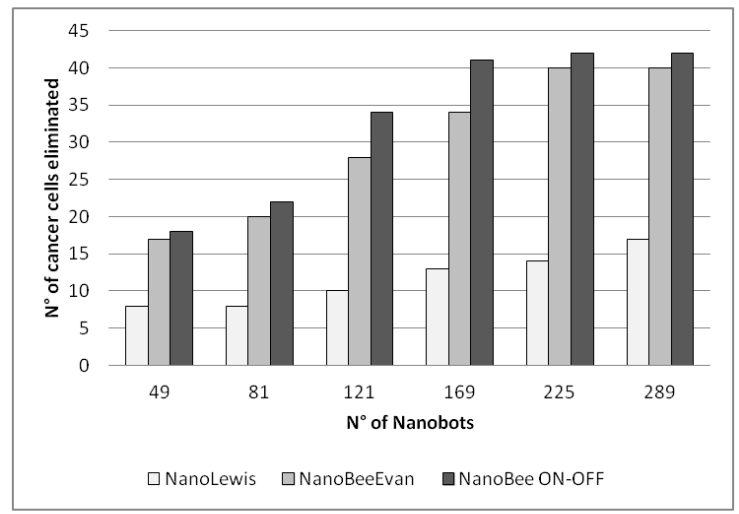

Figure 2: Number of eliminated cancer cells.

From the analysis conducted is clear that the algorithms considered with the associated parameters in terms of size of devices, available power, etc. are feasible for nanobots operating in the specific environment as we defined (i.e. the brain). When a different environment is considered, the characteristic parameters have to be re-defined and the effectiveness of the technique needs to be proven. In-vivo applications need a very accurate choice of available parameters.

\section{CONCLUSIONS AND FUTURE DIREC- TIONS}

In this work we evaluated the use of a swarm of nanobots in medical applications for treatment of endogenous diseases. We focused on the communication and organization aspects, necessary for the control of a single nanobot through very simple rules and based on only local information exchange. We took inspiration from a previous work where the behav- ior of the swarms of nanobots was defined through chemical signals able to attract the devices. After an accurate evaluation of the types of communication physically feasible for in vivo applications and based on the bees behavior in the supply phase, we proposed an algorithm based on acoustic signals, NanoBee. This choice is supported from the usual use of ultrasound waves for medical applications. By assuming the possibility for a nanobot to generate a vibration when it picks up a cellular tissue different from the normal tissue, we evaluated this approach and we compared the algorithms via simulation by a neural simulation tool, NSL. We showed that nano-bee techniques are more effective to face cancer than the Lewis'approach, by eliminating all the malignant cells. Concerning the future work there are many aspects that are worth to be considered in a deep way. The first one is concerning the chemical communication approach considered from Lewis and Bekey. In fact, during the simulation we were able to observing that the "barrier effect" worsens when CHEM-2 and CHEM-3 are activated. Maybe, an approach with only a single chemical substance would be more effective and above all in terms of nanobot design it is more feasible. Another important aspect is the validation of the probabilistic approach we adopted to simulate the attenuation of the signal with the square of the distance. It would be interesting to evaluating a real model of the brain and to compare it with this probabilistic approach.

\section{Acknowledgment}

This work has been carried out under the framework of STEM-Net, PRIN-National Italian Project \#H21J11000050001, financed by the Italian Ministry of University and Research.

\section{REFERENCES}

[1] B. Atakan and O. B. Akan. Body area nanonetworks with molecular communications in nanomedicine. IEEE Communications Magazine, 50(1):28-34, January 2012.

[2] A. Cavalcanti, B. Shirinzadeh, M. Zhang, and L. Kretly. Nanorobot hardware architecture for medical defense. ACM Trans. Program. Lang. Syst., 8(5):2932-2958, May 2008.

[3] M. Davis, J. Zuckerman, C. Choi, D. Seligson, A. Tolcher, C. A. abd J. Heidel, and A. Ribas. Nanorobots in brain tumor. Internation Research Journal of Pharmacy, 2(2):53-63, February 2011.

[4] M. Dorigo. Optimization, Learning and Natural Algorithms. PhD Thesis, Italy, 1992.

[5] R. Freitas. Nanomedicine, Volume I: Basic Capabilities. Landes Bioscience, 1999.

[6] T. Hog and R. F. Jr. Acoustic communication for medical nanorobots. Nano Communication Networks, doi:10.1016/j.nancom.2012.02.002(2):1-38, February 2012.

[7] A. Lewis and G. Bekey. The behavioral self-organization of nanorobots using local rules. In IEEE/RSJ IROS Conference Proceedings, pages 1333-1338, July 1992.

[8] A. Weitzenfeld, M. Arbib, and A. Alexander. The Neural Simulation Language. The MIT Press, 2004. 